This document is the Accepted Manuscript version of a Published Work that appeared in final

form in the Journal of Agricultural and Food Chemistry, copyright @ American Chemical

Society after peer review and technical editing by the publisher. To access the final edited and

published work see http://pubs.acs.org/doi/abs/10.1021/acs.jafc.6b04182

J. Agric. Food Chem. 2017, 65: 190-198

\title{
Fate of Fusarium Toxins during Brewing
}

Katharina Habler ${ }^{ \pm}$, Cajetan Geissinger ${ }^{\ddagger}$, Katharina Hofer ${ }^{\S}$, Jan

Schüler $^{ \pm}$, Sarah Moghari ${ }^{ \pm}$, Michael Hess ${ }^{\S}$, Martina Gastl ${ }^{ \pm}$and

Michael Rychlik ${ }^{\mathrm{\perp}, *}$

${ }^{ \pm}$Chair of Analytical Food Chemistry, Technische Universität München, Alte Akademie 10,

85354 Freising, Germany

${ }^{ \pm}$Chair of Brewery and Beverage Technology, Technische Universität München,

Weihenstephaner Steig 20, 85354 Freising, Germany

§Phytopathology, Technische Universität München, Emil Ramann Str. 2, 85354 Freising,

Germany

* Corresponding Author.

Phone: + 498161713153.

Fax: + 498161714216

E-Mail: michael.rychlik@tum.de.

Feldfunktion geändert

ORCID: 0000-0002-5826-6288 


\section{ABSTRACT}

Some information are available about the fate of Fusarium toxins during the brewing process, but only little is known about the single processing steps in detail. In our study we produced beer from two different barley cultivars inoculated with three different Fusarium species, namely Fusarium culmorum, Fusarium sporotrichioides, and Fusarium avenaceum, producing a wide range of mycotoxins such as type B trichothecenes, type A trichothecenes, and enniatins. By the use of multi-mycotoxin LC-MS/MS stable isotope dilution methods we were able to follow the fate of Fusarium toxins during the entire brewing process. In particular, the type B trichothecenes deoxynivalenol, 3-acetyldeoxynivalenol and 15-acetyldeoxynivalenol showed a similar behavior. Between 35 to $52 \%$ of those toxins remained in the beer after filtration. The contents of the potentially hazardous deoxynivalenol-3-glucoside and the type $A$ trichothecenes increased during mashing, but a rapid decrease of deoxynivalenol-3-glucoside content was found during the following steps of lautering and wort boiling. Concentration of enniatins greatly decreased with the discarding of spent grains or finally with the hot break. The results of our study show the retention of diverse Fusarium toxins during the brewing process and allow for assessing the food safety of beer regarding the monitored Fusarium mycotoxins.

KEYWORDS: Fusarium mycotoxins; stable isotope dilution assay; LC-MS/MS; barley; brewing process 


\section{INTRODUCTION}

With an average cultivation area of 50 million hectare and a crop size of 140 million tons worldwide in 2015 , barley can be rated along with wheat, rice and corn to the most important cereals in the world. ${ }^{1}$ Besides an increased production of cereals and barley, the worldwide beer output increased in the last ten years from 1.55 billion to 1.96 billion hectoliters in $2014 .^{2}$ Therefore, beer can be considered an important basic food. The quincentennial of the "German Beer Purity Law" and an average per capita beer consumption in Austria, Germany and the Czech Republic over 100 liters per person in $2014^{3}$ additionally underlines the need to ensure purity and quality of beer. Beer is produced from natural cereal products and cereal crops are susceptible to fungal infection. Fusarium head blight is a devastating fungal disease of small grain cereals and in particular associated with F. graminearum, F. culmorum, and F. avenaceum. ${ }^{4}$ The infection of barley with Fusarium species leads to losses in yield and grade and hence can result in declining end-use quality. Besides technological problems during malting and brewing, a heavy fungal infestation of brewing barley is mostly accompanied by a distinct mycotoxin contamination. ${ }^{5,6}$ Food and feed heavily loaded with Fusarium mycotoxins present a serious health risk for humans and animals and compromise food safety. Therefore, impeccable barley and barley malt quality is crucial for the production of high-quality beer. The maximum limits for unprocessed cereals, being equivalent to brewing malt, are legislatively set at 1250 $\mu \mathrm{g} / \mathrm{kg}$ for deoxynivalenol and at $100 \mu \mathrm{g} / \mathrm{kg}$ for zearalenone. ${ }^{7}$ Maximum levels for type A trichothecenes are recommended but have not yet been established.

The most abundant Fusarium toxins already observed in commercial beers are zearalenone, the type B trichothecenes nivalenol, deoxynivalenol-3-glucoside, 
deoxynivalenol, 3-acetyldeoxynivalenol and 15-acetyldeoxynivalenol (Figure 1). ${ }^{8-10}$ The so-called modified mycotoxin deoxynivalenol-3-glucoside ${ }^{11}$ that is mostly formed during germination due to high enzymatic activity of deoxynivalenolglycosyltransferase is an emerging issue concerning food safety and risk assessments. Deoxynivalenol-3-glucoside can be cleaved to deoxynivalenol in human and animal gastrointestinal tracts and thus can increase the initial amount of deoxynivalenol. ${ }^{12,13}$

Earlier studies focused on fermentation and provide little information about the influences of other key steps of the brewing process, especially mashing or lautering, on the fate of Fusarium toxins. We applied the newly developed multi-mycotoxin stable isotope dilution LC-MS/MS methods for cereals and beers ${ }^{14,10}$ to monitor the fate of Fusarium toxins (Figure 1) (deoxynivalenol; deoxynivalenol-3-glucoside; 3acetyldeoxynivalenol; 15-acetyldeoxynivalenol; HT-2-toxin; T-2-toxin; enniatin B, B1, A1, A; and beauvericin) throughout mashing and brewing. Our study was based on defined Fusarium species-specific contaminated basic material. Plants of two different barley varieties ('Grace' and 'Scarlett') were artificially infected in field trials with spores of three different Fusarium species (F. culmorum, F. sporotrichioides and F. avenaceum, respectively). Resulting raw grain was malted as previously reported. ${ }^{15}$ Subsequently, these barley malts were brewed on a pilot scale. Samples were taken at every key step of the brewing process. This study aimed at simultaneously monitoring the fate of ten Fusarium mycotoxins throughout the brewing process from barley malt grist to beer. 


\section{MATERIALS AND METHODS}

\section{Chemicals and reagents.}

Acetonitrile, methanol, and water (analytical grade) were purchased from VWR (Ismaning, Germany). Formic acid (>95\%) was bought from Sigma-Aldrich (Steinheim, Germany). The unlabeled reference compounds (deoxynivalenol-3glucoside, deoxynivalenol, 3-acetyldeoxynivalenol, 15-acetyldeoxynivalenol, HT-2toxin, T-2-toxin and some labeled standards $\left(\left[{ }^{13} \mathrm{C}\right]_{15}\right.$-deoxynivalenol, $\left[{ }^{13} \mathrm{C}\right]_{22}$-HT-2toxin) were bought from Coring System Diagnostix (Gernsheim, Germany). Enniatin B was obtained from Bioaustralis (New South Wales, Australia) and the other enniatins B1, A1 and A from Enzo Life Sciences (Lörrach, Germany). The labeled internal standards $\left(\left[{ }^{13} \mathrm{C}\right]_{2}\right.$-3-acetyldeoxynivalenol, $\left[{ }^{13} \mathrm{C}\right]_{4}$-T-2-toxin, $\left[{ }^{15} \mathrm{~N}\right]_{3}$-enniatin $\mathrm{B}$, $\left[{ }^{15} \mathrm{~N}\right]_{3}$-enniatin B1, $\left[{ }^{15} \mathrm{~N}\right]_{3}$-enniatin $\mathrm{A} 1$, and $\left[{ }^{15} \mathrm{~N}\right]_{3}$-enniatin $\mathrm{A}$ ) were synthesized in our laboratory as reported previously. ${ }^{16-18}$

\section{Raw material.}

Brewing experiments were carried out based on the same material as published recently. ${ }^{15}$ In brief, field experiments were conducted cultivating the spring barley varieties 'Grace' (Gr) and 'Scarlett' (Sc) in 2014. At the time period of anthesis, field plots $\left(11.25 \mathrm{~m}^{2}\right)$ were artificially infected with Fusarium species-specific conidial suspensions. Individual inocula were mixtures of spores from different isolates of $F$. culmorum (Fc, Fc002, Fc06, Fc03), F. avenaceum (Fa, Fa002, Fa01) and F. sporotrichioides (Fs, Fs001, Fs002, Fs03). Spore densities were dependent on individual spore production potential of strains and, therefore, different for each 
mixture (min.: $6.4 \times 10^{6}$ conidia/ $\mathrm{m}^{2}$, max.: $9.3 \times 10^{6}$ conidia $/ \mathrm{m}^{2}$ ). A respective number of plots remained non-inoculated and served as control. Plant protection treatments and fertilization were carried out according to regional standard rates. After harvesting matured plants by using a single plot combine, obtained raw grain material was stored in jute bags at room temperature in a dark and dry place. According to the standard MEBAK ${ }^{19}$ procedure and as already published ${ }^{15}$ the malting process was performed: steeping and germination time 6 days, germination temperature $15{ }^{\circ} \mathrm{C}$, $95 \%$ relative humidity, steeping degree $45 \%$, withering of germinated barley grains at $50{ }^{\circ} \mathrm{C}$ for $16 \mathrm{~h}$, then kilning at $60^{\circ} \mathrm{C}$ for $1 \mathrm{~h}$, at $70{ }^{\circ} \mathrm{C}$ for $1 \mathrm{~h}$, and finally at $80^{\circ} \mathrm{C}$ for 5 $\mathrm{h}$, followed by removing the rootlets at the end of kilning).

\section{Brewing process.}

Brewing was performed in a $10 \mathrm{~L}$ micro scale brewhouse at the Chair of Brewing and Beverage Technology (TUM). Before mashing in, the malt was ground in a two-rollmill. The adjusted distance between crushing rollers was $1.8 \mathrm{~mm}$. Grists $(\sim 1.5 \mathrm{~kg}$ each) were added to $6 \mathrm{~L}$ of distilled water $\left(62{ }^{\circ} \mathrm{C}\right)$. First rest was held on $62{ }^{\circ} \mathrm{C}$ with continuous stirring. Afterwards mash was heated up to $72{ }^{\circ} \mathrm{C}\left(1{ }^{\circ} \mathrm{C} / \mathrm{min}\right)$ and rest took another 30 min while stirring. When the second rest was finished, mash was heated up to $76{ }^{\circ} \mathrm{C}$ and the temperature was held for 5 min at $76{ }^{\circ} \mathrm{C}$. Afterwards mash was transferred in a preheated $\left(76^{\circ} \mathrm{C}\right)$ lauter tun, in which solid-liquid separation was done. First wort was lautered by following two additions of $1.5 \mathrm{~L}$ of pre-heated distilled water $\left(76{ }^{\circ} \mathrm{C}\right)$ for recausticizing spent grains. After lautering, wort was transferred into the wort kettle and boiled for $1 \mathrm{~h}$. Hops were added (calc. as 20 IBU in finished beer) when boiling started and wort boiling took another for 60 min until 
the original extract of $11.5 \% \mathrm{w} / \mathrm{w}$ was reached. Hot trub was removed in a whirlpool followed by wort cooling to $8{ }^{\circ} \mathrm{C}$ and pitching in a Cornelius container. The utilized yeast was a Fermentis Saflager 34/70. Main fermentation was carried out at a temperature of $12{ }^{\circ} \mathrm{C}$ till a gravity of $3.5 \% \mathrm{w} / \mathrm{w}$ was reached. Afterwards green beer was hosed (transferred in a new Cornelius container) and maturation followed for 4 days. Beer (around $8 \mathrm{~L}$ each) was cold stored for 3 weeks $\left(1{ }^{\circ} \mathrm{C}\right)$ and finally filtered with depth filter sheets (Seitz K 200).

\section{Separate mashing and lautering process.}

The laboratory scale mashing and lautering process was performed with three different malts of the variety 'Grace': The source material inoculated with $F$. culmorum that has already been used in the brewing process was used directly and further designated as "infected malt". Subsamples of this material were additionally autoclaved $\left(136{ }^{\circ} \mathrm{C}, 80 \mathrm{~min}, 3 \mathrm{bar}\right)$ to inactivate barley and fungal enzymes and later named "autoclaved malt". A so-called "mixed material" was prepared from $3 \mathrm{~g}$ autoclaved grist and $7 \mathrm{~g}$ non-inoculated control grist to exclude fungal contamination and to retain barley enzyme activities.

Ten $\mathrm{g}$ of each grist was mashed in with $40 \mathrm{~mL}$ distilled water $\left(62{ }^{\circ} \mathrm{C}\right)$ and the same mashing process and temperature program as described above was performed. For solid-liquid separation mash was transferred in a funnel with glass wool. Lautering was carried out by two additions of $10 \mathrm{~mL}$ pre-heated distilled water $\left(76{ }^{\circ} \mathrm{C}\right)$ for recausticizing spent grains. 


\section{Samples.}

Samples were taken at each key step during the brewing process. In detail, grist, mash, sweet wort, spent grains, original wort, hot break, green beer and finally filtered beer were sampled (Figure 2). Grist, mash, sweet wort, and spent grains were sampled during the laboratory scale mashing and lautering process. The total weight and the sampled weight were recorded for every sample. Mash, spent grains, and hot break were freeze-dried for $72 \mathrm{~h}$ before mycotoxin analysis and their water content was calculated. All results and concentrations of mycotoxins refer to the wet weight of the samples.

\section{Sample preparation.}

The sample preparations were performed as published recently. ${ }^{14,10}$ In brief, between $10 \mathrm{mg}$ and $1 \mathrm{~g}$ of finely ground homogenous solid samples (grist, freeze dried mash, spent grains, and hot break) had $10 \mathrm{~mL}$ acetonitrile/water (84:16, v/v) added. The samples were shaken in a laboratory shaker (225 rpm, 2 h). The resulting slurry was filtered and $4 \mathrm{~mL}$ of the filtrate was spiked with the internal standards $(50 \mu \mathrm{L}$ of $\left[{ }^{13} \mathrm{C}\right]_{15}$-deoxynivalenol $(1 \mu \mathrm{g} / \mathrm{mL}), 100 \mu \mathrm{L}$ of $\left[{ }^{13} \mathrm{C}\right]_{2}$-3-acetyldeoxynivalenol $(1 \mu \mathrm{g} / \mathrm{mL})$, $30 \mu \mathrm{L}$ of $\left[{ }^{13} \mathrm{C}\right]_{22}-\mathrm{HT}$-2-toxin $(1 \mu \mathrm{g} / \mathrm{mL}), 30 \mu \mathrm{L}$ of $\left[{ }^{13} \mathrm{C}\right]_{4}-\mathrm{T}-2$-toxin $(1 \mu \mathrm{g} / \mathrm{mL})$ and $80 \mu \mathrm{L}$ each of $\left[{ }^{15} \mathrm{~N}\right]_{3}$-enniatin $\mathrm{B},\left[{ }^{15} \mathrm{~N}\right]_{3}$-enniatin $\mathrm{B} 1,\left[{ }^{15} \mathrm{~N}\right]_{3}$-enniatin $\mathrm{A} 1$, and $\left[{ }^{15} \mathrm{~N}\right]_{3}$-enniatin $A$ $(0.1 \mu \mathrm{g} / \mathrm{mL})$. The filtrate volume was reduced when necessary to fall into the linear range of the response curves and to minimize standard consumption, but it was restocked to $4 \mathrm{~mL}$ with acetonitrile/water $(84: 16, \mathrm{v} / \mathrm{v})$ before purification. 
188 To $5 \mathrm{~mL}$ of degassed liquid samples (sweet wort, original wort, green beer, and 189 filtrated beer), $10 \mathrm{~mL}$ acetonitrile and the internal standards were added (50 $\mu \mathrm{L}$ of $190\left[{ }^{13} \mathrm{C}\right]{ }_{15}$-deoxynivalenol $(1 \mu \mathrm{g} / \mathrm{mL}), 150 \mu \mathrm{L}$ of $\left[{ }^{13} \mathrm{C}\right]_{2}$-3-acetyldeoxynivalenol $(1 \mu \mathrm{g} / \mathrm{mL})$, $19160 \mu \mathrm{L}$ of $\left[{ }^{13} \mathrm{C}\right]_{22}-\mathrm{HT}$-2-toxin $(1 \mu \mathrm{g} / \mathrm{mL}), 40 \mu \mathrm{L}$ of $\left[{ }^{13} \mathrm{C}\right]_{4}-\mathrm{T}$-2-toxin $(1 \mu \mathrm{g} / \mathrm{mL})$ and $70 \mu \mathrm{L}$ 192 of $\left[{ }^{15} \mathrm{~N}\right]_{3}$-enniatin $\mathrm{B},\left[{ }^{15} \mathrm{~N}\right]_{3}$-enniatin $\mathrm{B} 1,\left[{ }^{15} \mathrm{~N}\right]_{3}$-enniatin $\mathrm{A} 1$, and $\left[{ }^{15} \mathrm{~N}\right]_{3}$-enniatin $\mathrm{A}$, respectively $(0.1 \mu \mathrm{g} / \mathrm{mL})$. Deoxynivalenol-3-glucoside was quantitated using $\left[{ }^{13} \mathrm{C}\right]_{15^{-}}$ deoxynivalenol in liquid samples. The mixture was vortexed $(20 \mathrm{sec})$ and centrifuged (4000 rpm, $5 \mathrm{~min}, \mathrm{RT}$ ). The residue was extracted twice with $3 \mathrm{~mL}$ acetonitrile/water $(70: 30, v / v)$, respectively, and the combined supernatants were evaporated to dryness. Before purification the residue was dissolved in $4 \mathrm{~mL}$ acetonitrile/water $(84: 16, v / v)$.

The solid phase extraction was performed equally for solid and liquid samples. The samples were completely applied on a Bond Elut Mycotoxin cartridge, $500 \mathrm{mg}, 3 \mathrm{~mL}$, (Agilent Technologies, Santa Clara, CA) and the liquids were passed through the cartridges by vacuum suction. The eluates were collected and evaporated until dryness. The samples were reconstituted with $200 \mu \mathrm{L}$ acetonitrile/water $(1: 1, \mathrm{v} / \mathrm{v})$ and membrane filtered $(0.45 \mu \mathrm{m})$. Afterwards, LC-MS/MS analysis was performed.

Analog solid samples free of the monitored mycotoxins $(1 \mathrm{~g})$ were chosen as blank matrix for the matrix-matched calibration. The solid blank samples were spiked with six different amounts of deoxynivalenol-3-glucoside $(20-500 \mu \mathrm{gg} / \mathrm{kg})$. After evaporation of the solvent the same sample preparation and purification for solids was performed as described above. 
212 To follow the fate of the monitored Fusarium toxins during mashing and brewing 213 HPLC and MS/MS parameters of the previously published multi-mycotoxin LCMS/MS stable isotope dilution methods were used. ${ }^{14,10}$

In brief, LC-MS/MS was carried out on a Shimadzu LC-20A Prominence system (Shimadzu, Kyoto, Japan) with a $150 \times 3.0 \mathrm{~mm}$ ID, S-3 $\mu \mathrm{m}$ Hydrosphere RP-C 18 column (YMC Europe $\mathrm{GmbH}$, Dinslaken, Germany) and a $\mathrm{C}_{18}$-guard column (Phenomenex, Aschaffenburg, Germany) as stationary phase that was kept at $40^{\circ} \mathrm{C}$. The binary gradient system consisted of $(A) 0.1 \%$ formic acid and $(B)$ methanol with $0.1 \%$ formic acid at a flow rate of $0.2 \mathrm{~mL} / \mathrm{min}$. The gradient for the negative $\mathrm{ESI}$ mode was started and held at 10\% B for 2 min, raised linearly from $10-99 \%$ B during the next $6 \mathrm{~min}$, and then maintained at $99 \%$ B for $7.5 \mathrm{~min}$. Next, the mobile phase returned to $10 \%$ B within 2 min and the system was equilibrated for 9.5 min before the next run. The gradient for the positive ESI mode was started and held at $10 \% \mathrm{~B}$ for $2 \mathrm{~min}$, raised linearly from $10-87 \%$ B during the next $6 \mathrm{~min}$, held at $87 \%$ for $7 \mathrm{~min}$, raised to $100 \% \mathrm{~B}$ during the next $5 \mathrm{~min}$, and then maintained at $100 \% \mathrm{~B}$ for $3.5 \mathrm{~min}$. Next, the mobile phase returned to $10 \%$ B within 2 min and the system was equilibrated for $9.5 \mathrm{~min}$ before the next run. The injection volume was $10 \mu \mathrm{L}$.

The LC was interfaced with an API 4000 Qtrap hybrid triple quadrupole/linear ion trap mass spectrometer (Applied Biosystems Inc., Foster City, CA). It operated in the negative ESI mode for the analyte deoxynivalenol-3-glucoside in solid samples and in the positive ESI mode for the analytes deoxynivalenol-3-glucoside in liquid samples as well as deoxynivalenol, 3-acetyldeoxynivalenol, 15-acetyldeoxynivalenol, 
235 properties. The ion source parameters for the negative mode were set as follows:

236 curtain gas $20 \mathrm{psi}, \mathrm{CAD}$ gas pressure medium, ion spray voltage $-4500 \mathrm{eV}$, spray 237 gas $50 \mathrm{psi}$, dry gas $65 \mathrm{psi}$, and temperature $525^{\circ} \mathrm{C}$. The ion source parameters for 238 the positive mode were set as follows: curtain gas $20 \mathrm{psi}, \mathrm{CAD}$ gas pressure high, ion 239 spray voltage $4500 \mathrm{eV}$, spray gas $80 \mathrm{psi}$, dry gas $75 \mathrm{psi}$, and temperature $450{ }^{\circ} \mathrm{C}$. 


\section{RESULTS AND DISCUSSION}

\section{Sample preparation and LC-MS/MS analysis.}

The recently published multi-mycotoxin stable isotope dilution LC-MS/MS methods for cereals and beers ${ }^{14,10}$ were applied to follow the fate of ten Fusarium toxins throughout the brewing process. In detail, deoxynivalenol, deoxynivalenol-3glucoside, 3-acetyldeoxynivalenol, 15-acetyldeoxynivalenol, HT-2-toxin, T-2-toxin, enniatin $B$, enniatin $B 1$, enniatin $A 1$, and enniatin $A$ were analyzed in the samples at the following key steps of the brewing process (Figure 2): grist, mash, sweet wort, spent grains, original wort, hot break, green beer, and filtered beer. In the separate laboratory scale mashing and lautering process deoxynivalenol, deoxynivalenol-3glucoside, and the acetylated deoxynivalenol derivatives were determined in grist, mash, spent grains, and sweet wort of autoclaved, mixed, and inoculated malt grist, respectively. The contents of 15-acetyldeoxynivalenol in sweet wort, original wort, green beer, and beer were corrected for $72 \%$ recovery, according to the validation results recently published. ${ }^{10}$

\section{Fate of Fusarium toxins during brewing.}

Our brewing trials were based on the above described six inoculated and two control barley malts. In previous studies ${ }^{15}$ these raw materials were malted according to the standard MEBAK procedure. ${ }^{19}$ Plants for the control barley samples were grown under natural field conditions without inoculation. The mycotoxin levels of these control samples collected throughout brewing were mostly below the limit of detection or quantitation (data not shown). Hops and yeast added during brewing were also 
analyzed and classified as free from the monitored mycotoxins. Hence, in the present samples, all relevant toxin contents derived from inoculation with individual Fusarium species.

The concentrations of deoxynivalenol, deoxynivalenol-3-glucoside, 3acetyldeoxynivalenol, and 15-acetyldeoxynivalenol in the samples collected throughout the brewing process from inoculated barley batches are shown in Table 1. The behavior of deoxynivalenol, 3-acetyldeoxynivalenol, and 15-acetyldeoxynivalenol was similar in both cultivars throughout the brewing process. After mashing, the residual amounts of the latter mycotoxins ranged between $76 \%$ (Gr-Fc) and $120 \%$ (Sc-Fc) of the initial levels. The lautering step reduced the contents of deoxynivalenol, deoxynivalenol-3-glucoside, 3-acetyldeoxynivalenol, and 15acetyldeoxynivalenol by a maximum of $13 \%(\mathrm{Sc}-\mathrm{Fc})$ through the separation of the spent grains. A similar amount of $14 \%$ deoxynivalenol in spent grains was observed by Inoue et al.. ${ }^{20}$ In sweet wort around $84 \%$ deoxynivalenol, 94\% 3acetyldeoxynivalenol, and 65\% 15-acetyldeoxynivalenol of the initial level (average of both cultivars) was observed. Lancova et al. ${ }^{21}$ obtained similar ranges (70-90\%) for deoxynivalenol and acetyldeoxynivalenol derivatives in sweet wort made from naturally contaminated and artificially infected raw material. After wort boiling, on average $0.6 \%$ of each type B trichothecene was removed with the hot break. In line with previous reports ${ }^{20-22}$, we observed that fermentation, maturing, and filtration did not change significantly the concentrations of deoxynivalenol, 3acetyldeoxynivalenol, and 15-acetyldeoxynivalenol in original wort, as well as in green beer, and beer. In contrast to this, Nathanail et al. ${ }^{23}$ reported a decrease of maximum $15 \%$ for deoxynivalenol during fermentation. The absolute contents of 
deoxynivalenol, 3-acetyldeoxynivalenol, and 15-acetyldeoxynivalenol were lower in beer than in green beer as a result of losing beer during the filtration step. On average for both cultivars, $46 \%$ deoxynivalenol, $49 \% 3$-acetyldeoxynivalenol, and $35 \% 15$-acetyldeoxynivalenol of the initial levels remained in filtered beer. Our results on the contents of the latter toxins in beer are lower by a factor of 2 compared to findings previously published by Lancova et al. ${ }^{21}$ and Schwarz et al.. ${ }^{24}$ By contrast, Kostelanska et al. ${ }^{22}$ observed an increase of deoxynivalenol of $195-365 \%$ in final beer. These authors assumed this to result from a release of bound mycotoxins due to physiochemical and enzymatic processes.

Similar to the already described type B trichothecenes, the modified mycotoxin deoxynivalenol-3-glucoside increased to maximum $120 \%$ of the initial level after mashing, independent of the cultivar. But in contrast to deoxynivalenol, 3acetyldeoxynivalenol, and 15-acetyldeoxynivalenol, the content of deoxynivalenol-3glucoside rapidly decreased during lautering beyond expectation: Sweet wort had only $26 \%$ deoxynivalenol-3-glucoside in cultivar 'Grace' and 23\% deoxynivalenol-3glucoside in cultivar 'Scarlett' of the respective initial amounts. The spent grains were only contaminated with maximum $3.5 \%$ deoxynivalenol-3-glucoside (Sc-Fc). To our knowledge, this change of deoxynivalenol-3-glucoside during lautering has not been reported before. Lancova et al. ${ }^{21}$ and Kostelanska et al. ${ }^{22}$ investigated deoxynivalenol-3-glucoside from malt grist to final beer in several intermediate steps, but not in mash. In their studies, an increase of deoxynivalenol-3-glucoside from grist to sweet wort between $350-1400 \%$ was observed and again referred to a release of bound toxins during mashing due to physiochemical and enzymatic processes. However, no direct relation between deoxynivalenol-3-glucoside in mash and sweet 
wort could be made. In our study hardly any fluctuations in the concentrations of deoxynivalenol-3-glucoside in sweet wort, original wort, green beer, and beer could be measured in both cultivars. About 1.3\% deoxynivalenol-3-glucoside were removed with the hot break from the remaining process (average of both cultivars). At the end $18 \%$ deoxynivalenol-3-glucoside (Gr-Fc) or 13\% deoxynivalenol-3-glucoside (Sc-Fc) were left in filtered beer. Hence, lautering emerged as the most relevant step during brewing for removal of deoxynivalenol-3-glucoside. To verify and understand the modification or degradation of deoxynivalenol-3-glucoside during lautering we performed a laboratory scale mashing and lautering process. An enzyme based modification of deoxynivalenol-3-glucoside could be assumed because optimal temperatures for malt enzymes occurred during mashing. To investigate this assumption in more detail, in the separate laboratory scale mashing and lautering trial autoclaved malt was further used to exclude any enzyme activities of fungi and barley. Mixed malt (a mixture of autoclaved malt and control malt) was used to exclude fungal enzyme activity and retain barley enzyme activity. As repetition, the same malt inoculated with F. culmorum as already used in the brewing process was investigated to confirm our previous results.

We measured fungal and plant DNA of the infected, mixed and autoclaved malt via quantitative polymerase chain reaction. As published recently, ${ }^{15}$ in contrast to the control malt the infected malt was heavily loaded with Fusarium spp. DNA. Neither plant nor fungal DNA was detectable in the autoclaved malt (data not shown). Therefore, we concluded that along with the DNA the malt and fungal enzymes were destroyed during autoclaving. 
The fate of deoxynivalenol, deoxynivalenol-3-glucoside, 3-acetyldeoxynivalenol, and 15-acetyldeoxynivalenol during mashing and lautering of autoclaved, mixed, and inoculated malt is shown in Figure 3. The behavior of deoxynivalenol, deoxynivalenol3-glucoside, 3-acetyldeoxynivalenol, and 15-acetyldeoxynivalenol in inoculated malt of the laboratory scale mashing and lautering process was quite similar to the results of the brewing process. Consequently, the rapid decrease of deoxynivalenol-3glucoside as well as the steady behavior of the other type B trichothecenes during lautering could be confirmed. However, during lautering of mixed malt the same phenomenon of deoxynivalenol-3-glucoside appeared: We observed deoxynivalenol3 -glucoside at $28 \%$ of the initial level in sweet wort, whereas only $2 \%$ was removed with the spent grains and the level of deoxynivalenol remained stable. In contrast to the latter results, deoxynivalenol-3-glucoside showed a different behavior during lautering of the autoclaved malt: of the initial levels, $43 \%$ in sweet wort and $35 \%$ in spent grains could be found. The sum of both percentages (78\% deoxynivalenol-3glucoside) could approximately reach the original content of deoxynivalenol-3glucoside in mash and revealed a significantly higher level (at a two-sided p level < 0.01 ) than in inoculated or mixed malt (performing a statistical significance t-test with $\mathrm{n}=2$ ). The content of deoxynivalenol in the autoclaved malt trial again remained constant. 3-acetyldeoxynivalenol and 15-acetyldeoxynivalenol could not be detected in autoclaved and mixed malt. Thus, during mashing and lautering a modification or degradation of deoxynivalenol-3-glucoside to deoxynivalenol and acetylated deoxynivalenol derivatives or vice versa appeared rather improbable regarding our results. Hence, the decrease of deoxynivalenol-3-glucoside during lautering could be traced to enzyme activity in barley. A modification of deoxynivalenol-3-glucoside to deoxynivalenol-oligo/poly-glucosides ${ }^{25}$, deoxynivalenol glutathione adducts ${ }^{26}$ or 
other reported biologically modified deoxynivalenol products, e.g. deoxynivalenol cysteine, cysteine-glycine, malonylglucoside and hexoside derivatives ${ }^{27}$, might be possible.

The concentrations of HT-2-toxin and T-2-toxin in the samples collected throughout the brewing process from Fs-inoculated barley batches are shown in Table 2. During mashing we observed an increase of HT-2-toxin and T-2-toxin to a maximum of $200 \%$ and $330 \%$ of the initial levels, respectively. This increase can either be attributed to a further production of toxins due to fungal activity or to a release of bound mycotoxins due to physical or enzymatic processes. The latter hypothesis was also suggested by Kostelanska et al. ${ }^{28}$. Independent of the cultivar, $150 \%$ HT-2-toxin of the initial level was found in sweet wort. The analyzed spent grains were moderately contaminated with $24 \%$ HT-2-toxin in cultivar 'Grace' and 14\% HT-2-toxin in cultivar 'Scarlett'. In accordance with Lancova et al., ${ }^{21}$ the spent grains were heavier loaded with T-2-toxin: 75\% T-2-toxin in cultivar 'Grace' and 47\% T-2-toxin in cultivar 'Scarlett'. The mass difference of T-2-toxin between mash and sweet wort could almost completely be attributed to spent grains. The absolute amount of $410 \mu \mathrm{g}$ (280\% of the respective initial level) T-2-toxin (Gr-Fs) and $140 \mu \mathrm{g}(230 \%)$ T-2-toxin (Sc-Fs) remained in sweet wort. Maximum 2.5\% HT-2-toxin (Sc-Fa) and 3.7\% T-2toxin (Sc-Fa) were separated from wort with the hot break. In accordance with Lancova et al. ${ }^{21}$ and Inoue et al., ${ }^{20}$ the respective concentrations of HT-2-toxin and T-2-toxin in original wort hardly differed or did not differ at all from those in green beer or filtered beer. Nathanail et al. ${ }^{23}$ provide contrasting results. They reported a decrease of HT-2-toxin and T-2-toxin up to $34 \%$ during fermentation. In our study, the 
level of type A trichothecenes in beer could be reduced neither by fermentation nor by maturing, storage or filtration. Finally, 95\% HT-2-toxin and 135\% T-2-toxin of the initial levels were retrieved in filtered beer (average of both cultivars).

The concentrations of enniatin $B, B 1, A 1$, and $A$ in the samples collected throughout the brewing process from inoculated barley batches are shown in Table 3. During brewing all four monitored enniatins showed a similar behavior irrespective of barley cultivar. As indicated previously, ${ }^{15}$ enniatins were again present in following descending order of concentration: enniatin B, B1, A1, and A. After mashing, we could observe just a slight change of enniatins between $80 \%$ and $130 \%$ of the initial levels in malt grist (Gr-Fa, Sc-Fa). The subsequent lautering could be deemed as the most important step during brewing to separate enniatins from the remaining process. The analyzed spent grains were highly contaminated with enniatins and between $60 \%$ enniatin $\mathrm{B}(\mathrm{Sc}-\mathrm{Fa}$ ) and $130 \%$ enniatin $\mathrm{A}(\mathrm{Gr}-\mathrm{Fa})$ of the initial levels were found in the spent grains. Vaclavikova et al. ${ }^{29}$ and Hu et al. ${ }^{30}$ reported similar results of enniatins in spent grains. Accordingly, maximum 4.7\% enniatin B (Gr-Fa) was found in sweet wort, whereas enniatin A could no longer be detected in sweet wort (Gr-Fa, Sc-Fa). The levels of enniatin B1 and A1 in sweet wort were in between with $1.7 \%(\mathrm{Gr}-\mathrm{Fa})$ to $2.8 \%$ (Sc-Fa). As expected and already published by $\mathrm{Hu}$ et al. ${ }^{30}$, the contents of enniatins resolved in sweet wort were analogous to their polarity in the following decreasing order: enniatin $\mathrm{B}, \mathrm{B} 1, \mathrm{~A} 1$, and $\mathrm{A}$. The remaining amounts of enniatins were mostly removed with the hot break, in which between $0.3 \%$ enniatin $A$ (Gr-Fa, Sc-Fa) and 2.8\% enniatin B (Gr-Fa) of the initial enniatin levels were found. The concentrations of enniatin A in original wort, green beer, and beer (Gr-Fa, Sc-Fa) 
were below the limit of detection. In Sc-Fa, enniatin B1 could not be evidenced in green beer and beer and enniatin A1 was even in original wort no longer detectable. The amount of enniatins was hardly influenced by fermentation, storage, and filtration. A maximum of $0.5 \%$ enniatin of the initial level ( $\mathrm{Gr}-\mathrm{Fa})$ remained in finished beer.

With this study we could follow the fates of ten Fusarium mycotoxins from malt grist to filtered beer during the barley brewing process based on material deriving from two field-inoculated and subsequently malted barley cultivars. In general, all investigated Fusarium toxins were not affected significantly by wort boiling, fermentation, maturing, storage or filtration. In our study, mashing and lautering proved to be the most important and interesting steps influencing mycotoxin levels during brewing. Except for deoxynivalenol-3-glucoside, the other type B trichothecenes did not reveal major changes during brewing and between $35 \%$ and $52 \%$ of the initial levels remained in finished beer. Interestingly, deoxynivalenol-3-glucoside disappeared by more than $90 \%$ during lautering performed with water pre-heated to $76{ }^{\circ} \mathrm{C}$. These results demonstrate that the (temperature) conditions during lautering maintain a residual enzyme activity in barley suggesting further enzymatic modification of deoxynivalenol-3-glucoside tocompounds for which reference substances are not yet available. . Due to the inconsistent behavior of deoxynivalenol-3-glucoside during the brewing process a potential future maximum limit for this compound will not be accessible from a calculation based on the level of $18 \%$ for normal lager as it is commonly applied for deriving the maximum permitted level for deoxynivalenol from malt. 
433 In this study we investigated the fate of Fusarium toxins during brewing of two

434 different barley cultivars 'Grace' and 'Scarlett'. 'Scarlett' showed a lower abundance of type A trichothecenes and enniatins in malt and final beer up to $60 \%$ and $35 \%$ of the respective amounts in 'Grace'. In contrast to this, the output load for 'Scarlett' with type B trichothecenes was up to $50 \%$ higher than for 'Grace', but we could observe a similar contamination level in final beer regardless of the cultivar. The retention or accumulation of the respective Fusarium toxins during brewing was independent of the two barley varieties and their output load. Therefore, the two brewing trials based on the cultivars 'Grace' and 'Scarlett' could be taken as repetitions to confirm the respective behavior of the toxins. A statement concerning resistances of barley cultivars in respect of Fusarium toxins cannot be drawn from our results. Trichothecene content in our inoculated samples by far exceeded (up to a factor of $\sim 200$ ) the levels reported for most naturally infected spring barley samples. ${ }^{5,6,15}$

The spent grains removed from the brewing process were highly contaminated with type A trichothecenes and especially with enniatins. For enniatins, concentrations reported here can be similarly found in natural infected barley. ${ }^{31,30}$

Hence, spent grains or draff often used as animal feed might present a serious health risk to livestock. In "Fusarium-years" an analysis of spent grains regarding Fusarium toxins is recommended to prevent feeding of highly mycotoxin-loaded fodder. As enniatins are completely removed with the spent grains or latest with the hot break, in finished beer the type A and type B trichothecenes can be considered as more relevant with respect to food safety. However, the beers brewed from our control barley malts that are comparable to commercial brewing malt contained neither type A nor type B trichothecenes in 2015. Additionally considering our recent study of 
457 Fusarium toxins in beers ${ }^{10}$, it appears that even regular consumption of beer only

458 moderately exposes consumers to Fusarium toxins. Although the contamination level 459 in beer can be classified as low in the year 2015, it is subject to significant annual 460 variations and hence has to be minimized in any case.

461 


\section{ABBREVIATIONS}

464 Fa, Fusarium avenaceum; Fc, Fusarium culmorum; Fs, Fusarium sporotrichioides;

465 Gr, Grace; Sc, Scarlett

466

467 ACKNOWLEDGEMENT

468 This project was supported by the Forschungskreis der Ernährungsindustrie e.V. 469 (FEl, Bonn), the AiF, the German Federal Ministry of Economic Affairs and Energy 470 (AiF-Project No.: $17221 \mathrm{~N}$ ), Wissenschaftsförderung der Deutschen Brauwirtschaft 471 e.V., and Wissenschaftliche Station für Brauerei in München e.V. The authors thank 472 the Bavarian Bio-MS Center for running the LC-MS instrument and Ralph 473 Hückelhoven for critical reading of the manuscript. 


\section{References}

(1) United States Department of Agriculture World Agricultural Production, November 2015. http://apps.fas.usda.gov/psdonline/circulars/production.pdf. (Accessed: 9

December 2015)

(2) Barth-Haas Group Der Barth-Bericht, 2014/2015. http://www.barthhaasgroup.com/images/pdfs/reports/2015/BarthReport_20142015_DE.pdf. (Accessed: 9 December 2015)

(3) The Brewers of Europe Beer statistics 2015 edition, October 2015. http://www.brewersofeurope.org/uploads/mycmsfiles/documents/publications/2015/statistics_2015_web_002.pdf. (Accessed: 9 December 2015)

(4) Parry, D. W.; Jenkinson, P.; McLeed, L. Fusarium ear blight (scab) in small grain cereals-a review, Plant Pathology. 1995, 44, 207-218.

(5) Nielsen, L. K.; Jensen, J. D.; Nielsen, G. C.; Jensen, J. E.; Spliid, N. H.; Thomsen, I. K.; Justesen, A. F.; Collinge, D. B.; Jørgensen, L. N. Fusarium head blight of cereals in Denmark: species complex and related mycotoxins, Phytopathology. 2011, 101, 960-969.

(6) Linkmeyer, A.; Hofer, K.; Rychlik, M.; Herz, M.; Hausladen, H.; Hückelhoven, R.; Hess, M. Influence of inoculum and climatic factors on the severity of Fusarium head blight in German spring and winter barley, Food Addit. Contam., Part A 2016, Chemistry, analysis, control, exposure \& risk assessment. 33, 489-499.

(7) Commission Regulation (EC) No 1881/2006 of 19 December 2006 setting maximum levels for certain contaminants in foodstuffs. Off. J. Eur. Union. 2006, L364.

(8) Kostelanska, M.; Hajslova, J.; Zachariasova, M.; Malachova, A.; Kalachova, K.; Poustka, J.; Fiala, J.; Scott, P. M.; Berthiller, F.; Krska, R. Occurrence of deoxynivalenol and its major conjugate, deoxynivalenol-3-glucoside, in beer and some brewing intermediates, J. Agric. Food Chem. 2009, 57, 3187-3194.

(9) Scott, P. M. Mycotoxins Transmitted into beer from contaminated grains during brewing, Journal of AOAC International. 1996, 79, 875-882.

(10) Habler, K.; Gotthardt, M.; Schüler, J.; Rychlik, M. Multi-mycotoxin stable isotope dilution LC-MS/MS method for fusarium toxins in beer, Food Chem. 2017, 218, 447454.

(11) Rychlik, M.; Humpf, H.-U.; Marko, D.; Dänicke, S.; Mally, A.; Berthiller, F.; Klaffke, H.; Lorenz, N. Proposal of a comprehensive definition of modified and other forms of mycotoxins including "masked" mycotoxins, Mycotoxin Res. 2014, 30, 197205.

(12) Nagl, V.; Schwartz, H.; Krska, R.; Moll, W.-D.; Knasmüller, S.; Ritzmann, M.; Adam, G.; Berthiller, F. Metabolism of the masked mycotoxin deoxynivalenol-3glucoside in rats, Toxicol. Lett. 2012, 213, 367-373. 
(13) Nagl, V.; Woechtl, B.; Schwartz-Zimmermann, H. E.; Hennig-Pauka, I.; Moll, W.D.; Adam, G.; Berthiller, F. Metabolism of the masked mycotoxin deoxynivalenol-3glucoside in pigs, Toxicol. Lett. 2014, 229, 190-197.

(14) Habler, K.; Rychlik, M. Multi-mycotoxin stable isotope dilution LC-MS/MS method for Fusarium toxins in cereals, Anal. Bioanal. Chem. 2016, 408, 307-317.

(15) Habler, K.; Hofer, K.; Geißinger, C.; Schüler, J.; Hückelhoven, R.; Hess, M.; Gastl, M.; Rychlik, M. Fate of Fusarium toxins during the malting process, J. Agric. Food Chem. 2016, 64, 1377-1384.

(16) Asam, S.; Rychlik, M. Synthesis of four carbon-13-labeled type A trichothecene mycotoxins and their application as internal standards in stable isotope dilution assays, J. Agric. Food Chem. 2006, 54, 6535-6546.

(17) Asam, S.; Rychlik, M. Quantitation of type B-trichothecene mycotoxins in foods and feeds by a multiple stable isotope dilution assay, Eur Food Res Technol. 2007, 224, 769-783.

(18) Hu, L.; Rychlik, M. Biosynthesis of $15 \mathrm{~N} 3$-labeled enniatins and beauvericin and their application to stable isotope dilution assays, J. Agric. Food Chem. 2012, 60, 7129-7136.

(19) Anger, H.-M.; Mitteleuropäische Brautechnische Analysenkommission. Brautechnische Analysenmethoden: Rohstoffe: Gerste, Rohfrucht, Malz, Hopfen und Hopfenprodukte; Selbstverlag der MEBAK: Freising-Weihenstephan, Germany, 2006. (20) Inoue, T.; Nagatomi, Y.; Uyama, A.; Mochzuki, N. Fate of mycotoxins during beer brewing and fermentation, Biosci. Biotechnol. Biochem. 2013, 77, 1410-1415.

(21) Lancova, K.; Hajslova, J.; Poustka, J.; Krplova, A.; Zachariasova, M.; Dostalek, P.; Sachambula, L. Transfer of Fusarium mycotoxins and 'masked' deoxynivalenol (deoxynivalenol-3-glucoside) from field barley through malt to beer, Food Addit Contam. 2008, 25, 732-744.

(22) Kostelanska, M.; Zachariasova, M.; Lacina, O.; Fenclova, M.; Kollos, A.-L.; Hajslova, J. The study of deoxynivalenol and its masked metabolites fate during the brewing process realised by UPLC-TOFMS method, Food Chem. 2011, 126, 18701876.

(23) Nathanail, A. V.; Gibson, B.; Han, L.; Peltonen, K.; Ollilainen, V.; Jestoi, M.; Laitila, A. The lager yeast Saccharomyces pastorianus removes and transforms Fusarium trichothecene mycotoxins during fermentation of brewer's wort, Food Chem. 2016, 203, 448-455.

(24) Schwarz, P.; Howard, H. Fate and development of naturally occurring Fusarium mycotoxins during malting and brewing, J. Am. Soc. Brew. Chem. 1995, 53, $121-$ 127.

(25) Zachariasova, M.; Vaclavikova, M.; Lacina, O.; Vaclavik, L.; Hajslova, J. Deoxynivalenol oligoglycosides. New "masked" Fusarium toxins occurring in malt, beer, and breadstuff, J. Agric. Food Chem. 2012, 60, 9280-9291. 
556 (26) Gardiner, S. A.; Boddu, J.; Berthiller, F.; Hametner, C.; Stupar, R. M.; Adam, G.; 557 Muehlbauer, G. J. Transcriptome analysis of the barley-deoxynivalenol 558 interaction: Evidence for a role of glutathione in deoxynivalenol detoxification, The 559 American Phytopathological Society. 2010, 23, 962-976.

560 (27) Kluger, B.; Bueschl, C.; Lemmens, M.; Michlmayr, H.; Malachova, A.; Koutnik, 561 A.; Maloku, I.; Berthiller, F.; Adam, G.; Krska, R.; Schuhmacher, R.; Bai, G.

562 Biotransformation of the mycotoxin deoxynivalenol in Fusarium resistant and 563 susceptible near isogenic wheat lines, PloS one. 2015, 10, 1-19.

564 (28) Kostelanska, M.; Zachariasova, M.; Dzuman, Z.; Hajslova, J.; Ehrenbergerova, 565 J.; Cerkal, R.; Vaculova, K.; Mikyska, A.; Psota, V. Fusarium mycotoxins in spring 566 barley and their occurrence within the technological chain barley-malt-beer, Kvasny 567 Prumysl. 2011, 57, 209-214.

568 (29) Vaclavikova, M.; Malachova, A.; Veprikova, Z.; Dzuman, Z.; Zachariasova, M.; 569 Hajslova, J. 'Emerging' mycotoxins in cereals processing chains: Changes of 570 enniatins during beer and bread making, Food Chem. 2013, 136, 750-757.

571 (30) Hu, L.; Gastl, M.; Linkmeyer, A.; Hess, M.; Rychlik, M. Fate of enniatins and 572 beauvericin during the malting and brewing process determined by stable isotope 573 dilution assays, LWT - Food Sci Technol. 2014, 56, 469-477.

574 (31) Linkmeyer, A.; Götz, M.; Hu, L.; Asam, S.; Rychlik, M.; Hausladen, H.; Hess, M.; 575 Hückelhoven, R. Assessment and introduction of quantitative resistance to Fusarium 576 head blight in elite spring barley, Phytopathology. 2013, 103, 1252-1259. 
579 Figure 1. Structure of Type B (1-4), Type A Trichothecenes (5, 6), and Enniatins (7-

580 10)

581 Figure 2. Key Steps of Brewing Process

582 Figure 3. Fate of Deoxynivalenol (1), Deoxynivalenol-3-Glucoside (2), 3-

583 Acetyldeoxynivalenol (3), and 15-Acetyldeoxynivalenol (4) during Mashing and

584 Lautering of (A) Autoclaved Malt, (B) Mixed Malt (a mixture of autoclaved and non-

585 inoculated control malt), and (C) Infected Malt (derived from field-inoculated and

586 subsequently malted barley)

587 


\section{Tables}

Table 1. Contents of Type B Trichothecenes in Grist, Mash, Sweet Wort, Spent Grains, Original Wort, Hot Break, Green Beer, and Beer Prepared from Two Barley Cultivars 'Grace' (Gr-Fc) and 'Scarlett' (Sc-Fc) Inoculated with F. culmorum

\begin{tabular}{|c|c|c|c|c|c|c|c|c|c|c|c|c|}
\hline \multirow[t]{3}{*}{ Sample ID } & \multicolumn{3}{|c|}{ DON (1) } & \multicolumn{3}{|c|}{ D3G (2) } & \multicolumn{3}{|c|}{ 3-AcDON (3) } & \multicolumn{3}{|c|}{ 15-AcDON (4) } \\
\hline & \multicolumn{3}{|c|}{ Total } & \multicolumn{3}{|c|}{ Total } & \multicolumn{3}{|c|}{ Total } & \multicolumn{3}{|c|}{ Total } \\
\hline & $\mu \mathrm{g} / \mathrm{kg}$ & $\mathbf{m g}$ & $\%$ & $\mu \mathrm{g} / \mathrm{kg}$ & $\mathrm{mg}$ & $\%$ & $\mu \mathrm{g} / \mathrm{kg}$ & $\mathrm{mg}$ & $\%$ & $\mu \mathrm{g} / \mathrm{kg}$ & $\mu \mathrm{g}$ & $\%$ \\
\hline \multicolumn{13}{|l|}{ Gr-Fc } \\
\hline Grist & 10000 & 9.3 & 100 & 19000 & 17 & 100 & 1800 & 1.6 & 100 & 670 & 600 & 100 \\
\hline Mash & 1700 & 7.3 & 78 & 4900 & 21 & 120 & 380 & 1.6 & 100 & 110 & 450 & 76 \\
\hline Sweet wort & 1500 & 7.8 & 84 & 830 & 4.3 & 26 & 290 & 1.5 & 93 & $68^{b}$ & 360 & 60 \\
\hline Spent grains & 240 & 0.2 & 2.5 & 510 & 0.5 & 2.9 & 63 & 0.06 & 3.8 & 55 & 54 & 9.1 \\
\hline Original wort & 1400 & 7.2 & 77 & 880 & 4.4 & 26 & 250 & 1.3 & 79 & $65^{\mathrm{b}}$ & 320 & 54 \\
\hline Hot break & 420 & 0.05 & 0.6 & 1900 & 0.2 & 1.4 & 68 & 0.01 & 0.5 & 21 & 4.6 & 0.5 \\
\hline Green beer & 1500 & 7.1 & 76 & 1100 & 4.9 & 29 & 260 & 1.2 & 76 & $67^{\mathrm{b}}$ & 310 & 52 \\
\hline Beer & 1400 & 4.6 & 50 & 980 & 3.3 & 18 & 260 & 0.8 & 52 & $64^{b}$ & 210 & 35 \\
\hline \multicolumn{13}{|l|}{ Sc-Fc } \\
\hline Grist & 13000 & 10 & 100 & 28000 & 22 & 100 & 2400 & 1.9 & 100 & 870 & 690 & 100 \\
\hline Mash & 2200 & 8.9 & 85 & 6500 & 26 & 120 & 530 & 2.1 & 110 & 200 & 800 & 120 \\
\hline Sweet wort & 1900 & 8.6 & 83 & 1100 & 5.0 & 23 & 390 & 1.8 & 94 & $110^{b}$ & 490 & 71 \\
\hline Spent grains & 300 & 0.3 & 2.8 & 800 & 0.8 & 3.5 & 98 & 0.1 & 5 & 93 & 89 & 13 \\
\hline Original wort & 1800 & 8.5 & 81 & 990 & 4.6 & 21 & 360 & 1.7 & 90 & $94^{\mathrm{b}}$ & 440 & 64 \\
\hline Hot break & 1000 & 0.1 & 0.7 & 3500 & 0.3 & 1.2 & 180 & 0.01 & 0.7 & 53 & 3.9 & 0.6 \\
\hline Green beer & 1900 & 8.3 & 80 & 1100 & 4.8 & 22 & 340 & 1.5 & 80 & $100^{b}$ & 440 & 64 \\
\hline Beer & 1500 & 4.3 & 42 & 980 & 2.9 & 13 & 300 & 0.9 & 46 & $82^{b}$ & 240 & 35 \\
\hline
\end{tabular}

${ }^{a}$ below limit of quantitation; ${ }^{\circ}$ recovery-corrected $(72 \%)$ 
Table 2. Contents of Type A Trichothecenes in Grist, Mash, Sweet Wort, Spent Grains, Original Wort, Hot Break, Green Beer, and Beer Prepared from Two Barley Cultivars 'Grace' (Gr-Fc) and 'Scarlett' (Sc-Fc) Inoculated with F. sporotrichioides

\begin{tabular}{|c|c|c|c|c|c|c|}
\hline \multirow{3}{*}{ Sample ID } & \multicolumn{3}{|c|}{ HT-2-toxin (5) } & \multicolumn{3}{|c|}{ T-2-toxin (6) } \\
\hline & \multirow[b]{2}{*}{$\mu \mathrm{g} / \mathrm{kg}$} & \multicolumn{2}{|c|}{ Total } & \multirow[b]{2}{*}{$\mu \mathrm{g} / \mathrm{kg}$} & \multicolumn{2}{|c|}{ Total } \\
\hline & & $\mu g$ & $\%$ & & $\mu g$ & $\%$ \\
\hline \multicolumn{7}{|l|}{ Gr-Fs } \\
\hline Grist & 180 & 120 & 100 & 200 & 130 & 100 \\
\hline Mash & 72 & 270 & 200 & 130 & 490 & 330 \\
\hline Sweet wort & 45 & 200 & 150 & 90 & 410 & 280 \\
\hline Spent grains & 36 & 32 & 24 & 120 & 110 & 75 \\
\hline Original wort & 38 & 170 & 120 & 72 & 320 & 210 \\
\hline Hot break & 38 & 2.2 & 1.6 & 50 & 2.9 & 2.0 \\
\hline Green beer & 42 & 170 & 130 & 79 & 320 & 220 \\
\hline Beer & 41 & 120 & 92 & 71 & 210 & 140 \\
\hline \multicolumn{7}{|l|}{ Sc-Fs } \\
\hline Grist & 69 & 53 & 100 & 78 & 61 & 100 \\
\hline Mash & 26 & 100 & 190 & 41 & 160 & 260 \\
\hline Sweet wort & 18 & 82 & 150 & 30 & 140 & 230 \\
\hline Spent grains & 7.9 & 7.7 & 14 & 29 & 28 & 47 \\
\hline Original wort & 15 & 67 & 130 & 25 & 110 & 180 \\
\hline Hot break & 22 & 1.3 & 2.5 & 37 & 2.3 & 3.7 \\
\hline Green beer & 16 & 64 & 120 & 26 & 107 & 180 \\
\hline Beer & 17 & 52 & 98 & 25 & 77 & 130 \\
\hline
\end{tabular}


Table 3. Contents of Enniatins in Grist, Mash, Sweet Wort, Spent Grains, Original Wort, Hot Break, Green Beer, and Beer Prepared from Two Barley Cultivars 'Grace' (Gr-Fc) and 'Scarlett' (Sc-Fc) Inoculated withF. avenaceum

\begin{tabular}{|c|c|c|c|c|c|c|c|c|c|c|c|c|}
\hline \multirow[t]{3}{*}{ Sample ID } & \multicolumn{3}{|c|}{ enniatin B (7) } & \multicolumn{3}{|c|}{ enniatin B1 (8) } & \multicolumn{3}{|c|}{ enniatin A1 (9) } & \multicolumn{3}{|c|}{ enniatin A (10) } \\
\hline & \multirow[b]{2}{*}{$\mu \mathrm{g} / \mathbf{k g}$} & \multicolumn{2}{|c|}{ Total } & \multirow[b]{2}{*}{$\mu \mathrm{g} / \mathrm{kg}$} & \multicolumn{2}{|c|}{ Total } & \multirow[b]{2}{*}{$\mu \mathrm{g} / \mathrm{kg}$} & \multicolumn{2}{|c|}{ Total } & \multirow[b]{2}{*}{$\mu \mathrm{g} / \mathrm{kg}$} & \multicolumn{2}{|c|}{ Total } \\
\hline & & $\mu \mathrm{g}$ & $\%$ & & $\mu g$ & $\%$ & & $\mu g$ & $\%$ & & $\mu \mathrm{g}$ & $\%$ \\
\hline \multicolumn{13}{|l|}{ Gr-Fa } \\
\hline Grist & 6100 & 4100 & 100 & 1200 & 820 & 100 & 160 & 100 & 100 & 12 & 8 & 100 \\
\hline Mash & 1200 & 3900 & 97 & 310 & 1000 & 120 & 42 & 140 & 130 & 3.1 & 10 & 130 \\
\hline Sweet wort & 38 & 160 & 4.7 & 4.7 & 20 & 2.4 & 0.4 & 1.8 & 1.7 & - & & \\
\hline Spent grains & 3800 & 3100 & 92 & 1100 & 940 & 110 & 150 & 120 & 120 & 12 & 10 & 130 \\
\hline Original wort & 9.0 & 35 & 1.0 & 3.4 & 13 & 1.6 & 0.5 & 1.8 & 1.8 & - & & \\
\hline Hot break & 2600 & 94 & 2.8 & 210 & 7.7 & 0.9 & 15 & 0.6 & 0.5 & 0.7 & 0.03 & 0.3 \\
\hline Green beer & 3.6 & 13 & 0.4 & 2.4 & 8.8 & 1.1 & 0.3 & 1.0 & 0.9 & - & & \\
\hline Beer & 2.6 & 6.9 & 0.2 & 1.4 & 3.7 & 0.5 & 0.2 & 0.5 & 0.5 & - & & \\
\hline \multicolumn{13}{|l|}{ Sc-Fa } \\
\hline Grist & 4200 & 3900 & 100 & 890 & 820 & 100 & 100 & 97 & 100 & 8.4 & 7.8 & 100 \\
\hline Mash & 720 & 3300 & 86 & 190 & 860 & 110 & 22 & 103 & 110 & 1.4 & 6.2 & 80 \\
\hline Sweet wort & 19 & 100 & 2.7 & 3.2 & 17 & 2.1 & $0.5^{\mathrm{a}}$ & 2.7 & 2.8 & - & & \\
\hline Spent grains & 2000 & 2300 & 60 & 560 & 640 & 78 & 65 & 75 & 78 & 5.3 & 6.1 & 78 \\
\hline Original wort & 4.5 & 24 & 0.6 & 0.2 & 1.3 & 0.2 & - & & & - & & \\
\hline Hot break & 1600 & 70 & 1.8 & 150 & 6.4 & 0.8 & 11 & 0.5 & 0.5 & 0.6 & 0.03 & 0.3 \\
\hline Green beer & 1.0 & 4.9 & 0.1 & - & & & - & & & - & & \\
\hline Beer & 0.9 & 2.6 & 0.07 & - & & & - & & & - & & \\
\hline
\end{tabular}




\section{Figure 1.}

\section{Type B Trichothecenes}

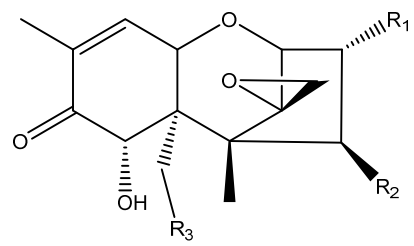

Deoxynivalenol, DON (1)

$\mathrm{R}_{1}=\mathrm{R}_{3}=\mathrm{OH}, \mathrm{R}_{2}=\mathrm{H}$,

Deoxynivalenol-3-glucoside, D3G (2) $\quad \mathrm{R}_{1}=\mathrm{Glc}, \mathrm{R}_{2}=\mathrm{H}, \mathrm{R}_{3}=\mathrm{OH}$

3-Acetyldeoxynivalenol, 3-AcDON (3) $\quad \mathrm{R}_{1}=\mathrm{OAc}, \mathrm{R}_{2}=\mathrm{H}, \mathrm{R}_{3}=\mathrm{OH}$

15-Acetyldeoxynivalenol, 15-AcDON (4) $R_{1}=R_{3}=O A c, R_{2}=H$

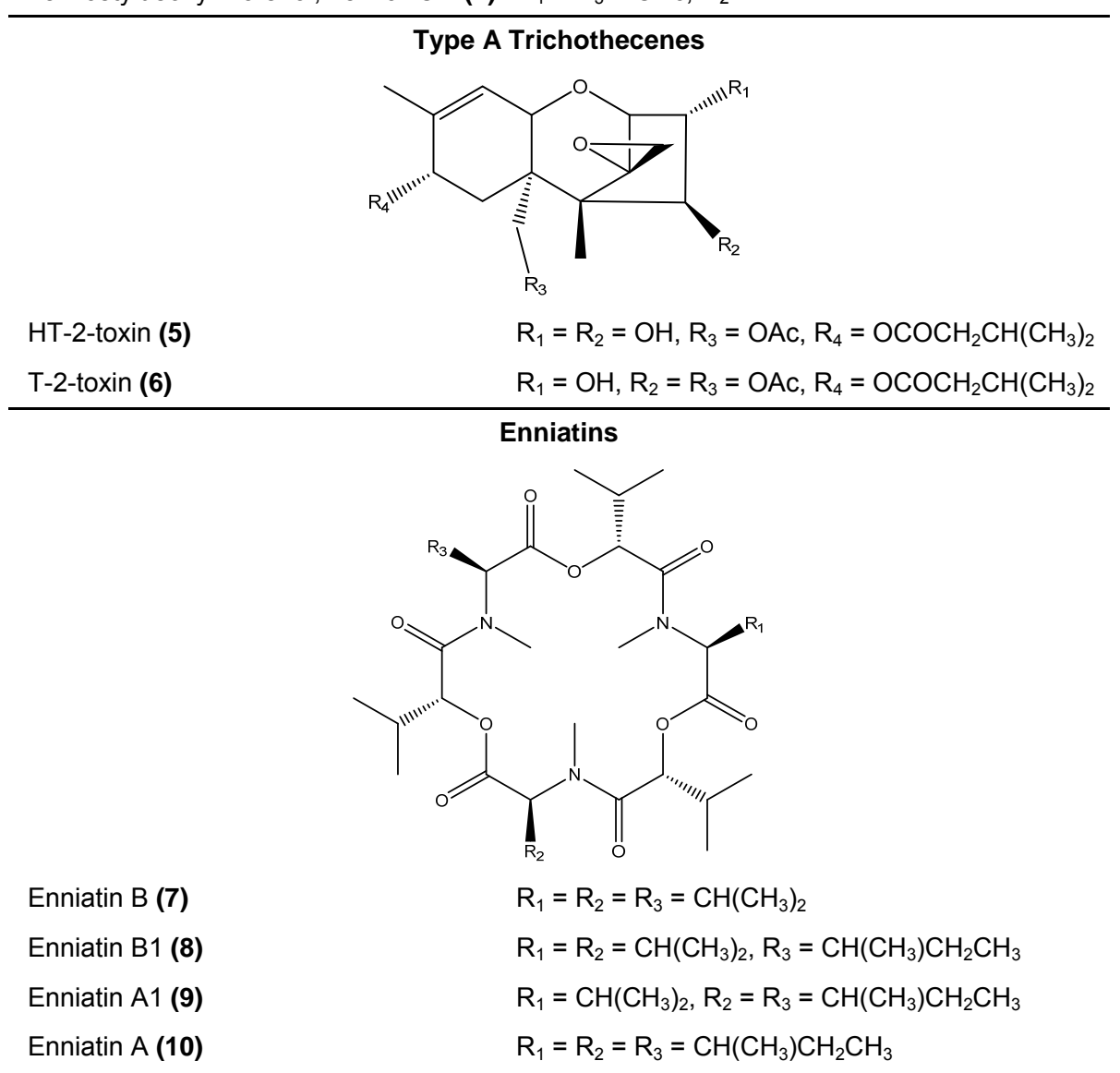


Figure 2.

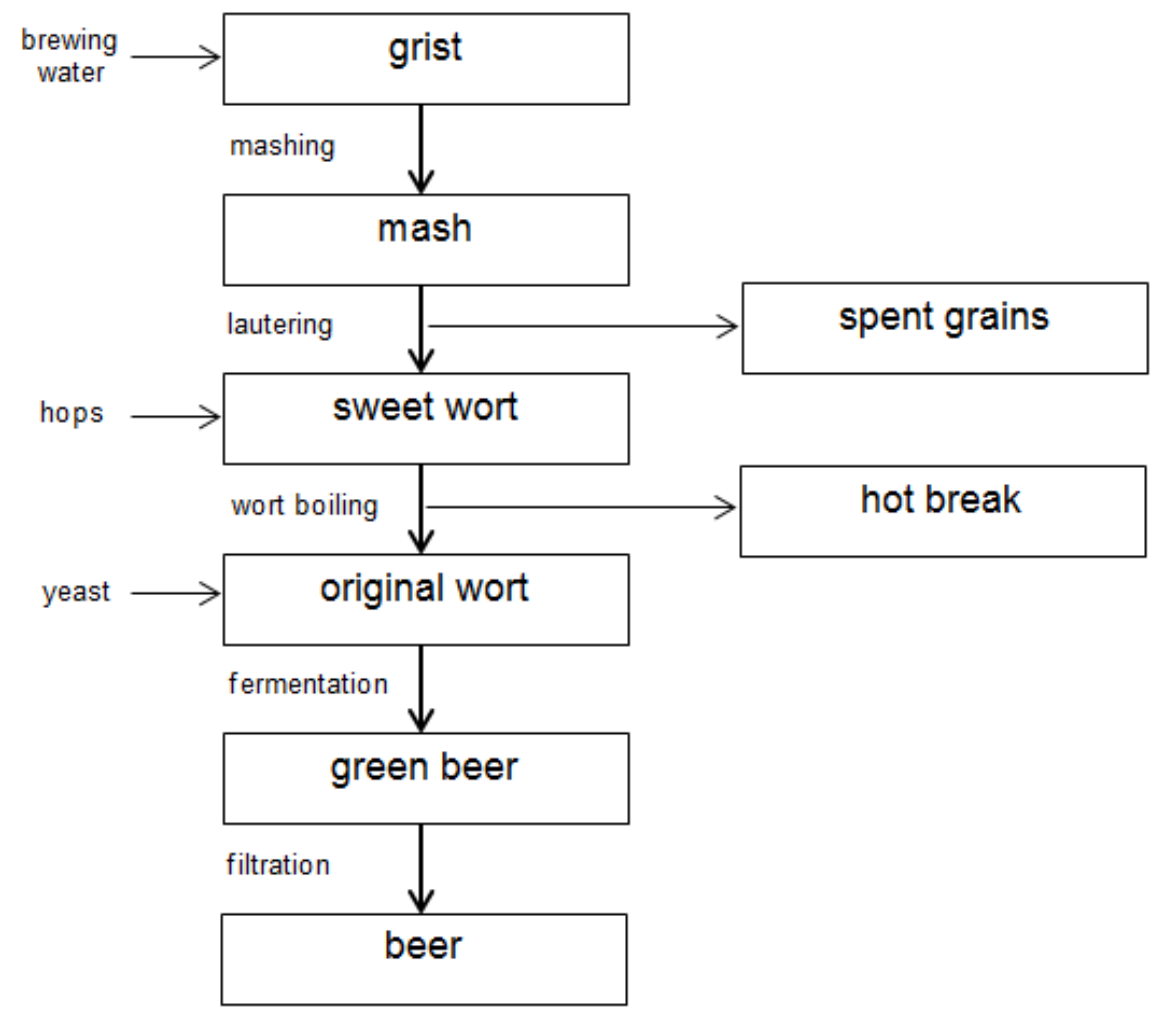


Figure 3.

\section{(A) Autoclaved Malt}

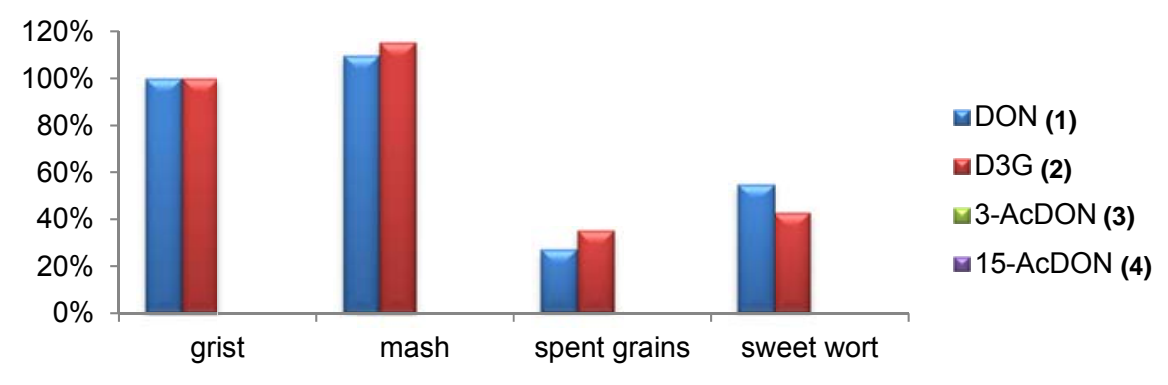

(B) Mixed Malt

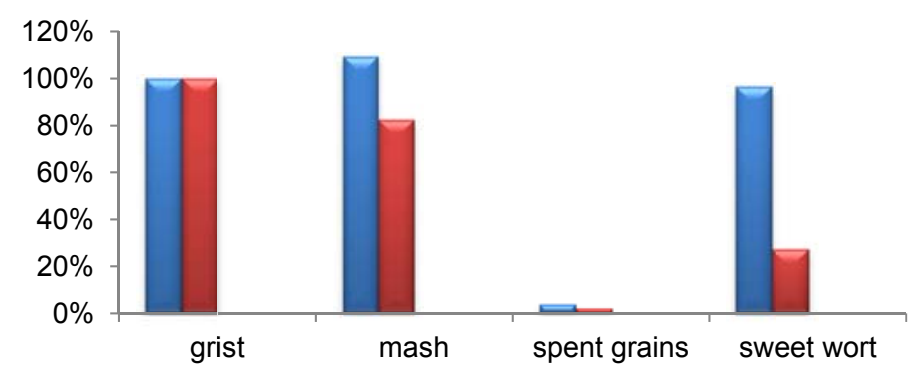

(C) Infected Malt

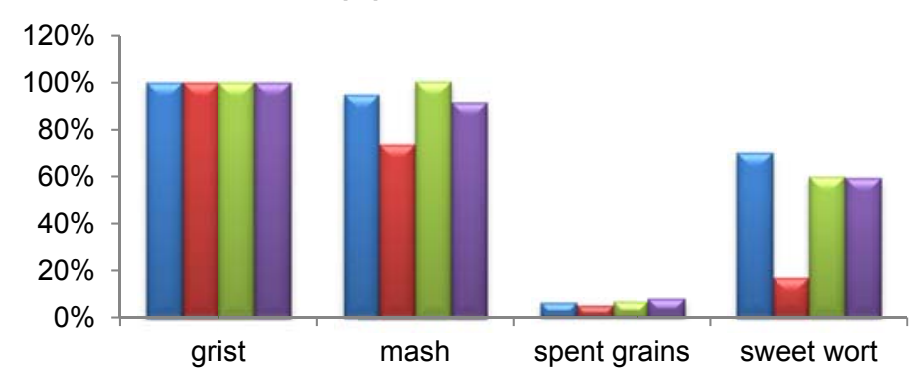


Table of Contents Graphic

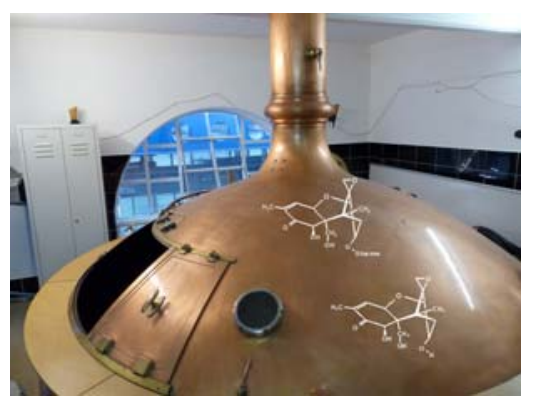

